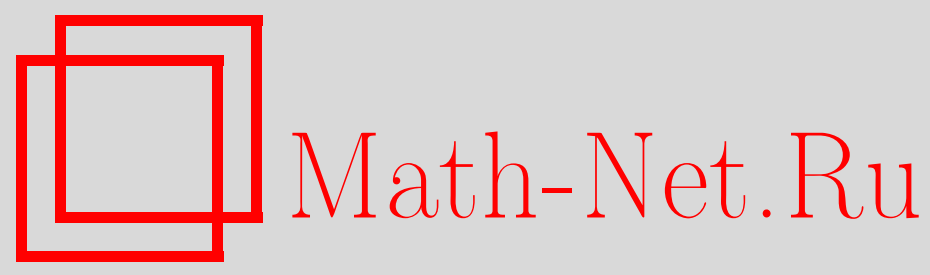

В. В. Филиппов, О существовании периодических решений, Матем. заметки, 1997, том 61, выпуск 5, 769-784

DOI: https://doi.org/10.4213/mzm1559

Использование Общероссийского математического портала Math-Net.Ru подразумевает, что вы прочитали и согласны с пользовательским соглашением http://www . mathnet.ru/rus/agreement

Параметры загрузки:

IP: 54.84 .234 .179

26 апреля 2023 г., 15:31:00

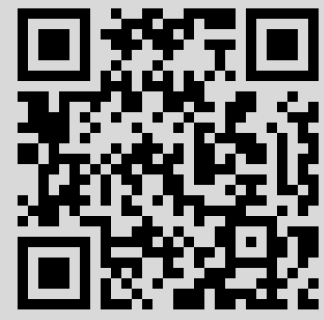




\section{О СУЩЕСТВОВАНИИ ПЕРИОДИЧЕСКИХ РЕШЕНИЙ}

\section{В.В. Филиппов}

Развивается аппарат доказательства теорем существования периодических решений для уравнений с разрьвной правой частью и дифференциальных включений.

Библиография: 9 названий.

В этой статье будет указана возможность расширения сферы применимости подхода [1] к вопросу существования периодических решений на более широкие классы обыкновенных дифференциальных уравнений и включений.

Мы воспользуемся идеями подхода [2]-[4]. Частью обозначений из [2] мы будем пользоваться без дополнительных пояснений (см. также [3], [4]).

Ранее аналоги наших результатов доказьвались для уравнений с непрерьвной правой частью либо для уравнений с правой частью, удовлетворяющей условиям Каратеодори. Наши результаты, благодаря возникающей в рамках нашего общего подхода теории задачи Коши (см. [2]-[5]), оказьваются применимьми к уравнениям и включениям со сложными разрьвами правых частей, в том числе, и по пространственным переменным.

\section{§1. О длине интервала существования решения задачи Коши}

Будем считать, что правая часть рассматриваемого дифференциального включения

$$
y^{\prime} \in F(t, y)
$$

определена на подмножестве $U=] a, b\left[\times \mathbb{R}^{n}\right.$ произведения $\mathbb{R} \times \mathbb{R}^{n}$.

В заметке [6] было указано достаточное условие единственности нулевого решения.

Отсутствие единственности нулевого решения - это возможность для решения $z(t)$, $t_{0} \leqslant t \leqslant t_{1}$, где $\left.\left[t_{0}, t_{1}\right] \subseteq\right] a, b\left[\right.$, подойти к нулевой точке $\overline{0} \in \mathbb{R}^{n}$ из области $\mathbb{R}^{n} \backslash\{\overline{0}\}$. При этом можно ограничиться рассмотрением включения (1) только в области $\left.U_{0}=\right] a, b[\times$ $\left(\mathbb{R}^{n} \backslash\{\overline{0}\}\right)$, и тогда отсутствие единственности нулевого решения для включения (1) в $U$ приобретает вид существования решения $z(t), t_{0} \leqslant t<t_{1}$, включения $(1)$, для которого $\|z(t)\| \rightarrow 0$ при $t \rightarrow t_{1}$, или решения $z(t), t_{0}<t \leqslant t_{1}$, для которого $\|z(t)\| \rightarrow 0$ при $t \rightarrow t_{0}$.

В такой постановке вопрос о единственности нулевого решения оказывается аналогичным вопросу о вьполнении следующего условия:

любое решение включения (1), область определения которого задевает отрезок

$\left[t_{0}, t_{1}\right]$ продолжается на весь отрезок $\left[t_{0}, t_{1}\right]$, 
ибо невьполнение этого условия (при естественных ограничениях на (1)) означает существование решения $z(t), t_{0} \leqslant t<t_{1}$, включения (1) в области $U_{0}$, для которого $\|z(t)\| \rightarrow \infty$ при $t \rightarrow t_{1}$, или решения $z(t), t_{0}<t \leqslant t_{1}$, для которого $\|z(t)\| \rightarrow \infty$ при $t \rightarrow t_{0}$.

Поэтому появляется возможность применить использованные в [6] соображения для исследования вопроса о длине интервала существования решения. Для упрощения формулировок мы не будем стремиться к максимальной общности.

Напомним некоторые основные обозначения из [2]-[4].

Символ $R^{i}(U)$ обозначает множество всех $Z \subseteq C_{s}(U)$, удовлетворяющих условию:

если $z \in Z$ и отрезок $I$ лежит в области определения $\pi(z)$ функции $z$, то $\left.z\right|_{I} \in Z$.

Символ $R(U)$ обозначает множество всех пространств $Z \in R^{i}(U)$, удовлетворяющих условию:

если $z_{1}, z_{2} \in Z, I=\pi\left(z_{1}\right) \cap \pi\left(z_{2}\right) \neq \varnothing$ и $\left.z_{1}\right|_{I}=\left.z_{2}\right|_{I}$, то функция

$$
z(t)= \begin{cases}z_{1}(t) & \text { при } t \in \pi\left(z_{1}\right), \\ z_{2}(t) & \text { при } t \in \pi\left(z_{2}\right)\end{cases}
$$

(определенная на отрезке $\pi\left(z_{1}\right) \cup \pi\left(z_{2}\right)$ ) принадлежит множеству $Z$.

Символ $s(U)$ обозначает множество всех последовательностей $\left\{Z_{i}: i=1,2, \ldots\right\} \subseteq R^{i}(U)$, удовлетворяющих условию:

для любого $K \in \exp _{c} U$ любая последовательность функций $z_{j} \in\left(Z_{i_{j}}\right)_{K}, j=1,2, \ldots$

$\left(i_{1}<i_{2}<\ldots\right)$, равностепенно непрерывна.

Символ $\langle M\rangle$ обозначает внутренность, а $[M]$ - замыкание множества $M$. При необходимости уточнить, относительно какого пространства они берутся, символ пространства помещается в виде нижнего индекса: $[M]_{X}$.

Замена переменных в чистом виде - это некоторый гомеоморфизм области определения правой части рассматриваемого уравнения. Если $f$ - такая замена, то $\widetilde{f}(z)$ обозначает образ решения $z$ при действии замены переменных $f$ (см. [6]).

ЛЕмма 1.1. Пусть $X$ - связный бикомпакт, $Y$ - его замкнутое подмножество. Тогда замыкание в $X$ любой компоненты связности множсества $X \backslash Y$ пересекается $с Y$.

ДокАЗАТЕЛьСтво. Сформулированное утверждение является прямым следствием леммы 6.1.25 в [7].

TEOPEMA 1.1. Пусть

1) $\left.r \geqslant 0, V_{r}=\left\{u: u \in \mathbb{R}^{n},\|u\|>r\right\}, U_{r}=\right] a, b\left[\times V_{r}, Z \in R^{i}\left(U_{r}\right)\right.$;

2) $\lambda_{1}>\lambda_{2}>\cdots, \lambda_{i} \rightarrow 0$ npu $i \rightarrow \infty, \psi_{i}(t, y)=\left(t, \lambda_{i} y\right)$;

3) $\gamma$ - семейство открытых подмножеств области $U_{r}$, для любого әлемента $V$ которого $\left\{\left(\widetilde{\psi}_{i}(Z)\right)_{V}: i=1,2, \ldots\right\} \in s(V)$

4) $\left.\left[t_{0}, t_{1}\right] \subseteq\right] a, b\left[u\right.$ для любой точки $t \in\left[t_{0}, t_{1}\right]$ все компоненты связности множества $\left(\{t\} \times V_{r}\right) \backslash \bigcup \gamma$ ограничены.

Тогда мнохсество значений любой функиии из множества $M=\left\{z: z \in Z^{-} \cup Z^{+}\right.$, $\left.\pi(z) \subseteq\left[t_{0}, t_{1}\right]\right\}$ является ограниченным. 
ДокАЗАТЕЛьСтво. Допустим, найдется такая функция $z \in M$, что

$$
\sup \{\|z(t)\|: t \in \pi(z)\}=\infty
$$

Положим для определенности, что $z \in Z^{+}$. Пусть $\pi(z)=\left[p, q\left[, \mu_{i}=1 / \lambda_{i}\right.\right.$.

Отметим последовательно индексы $\alpha(0)<\alpha(1)<\alpha(2)<\cdots$ и точки $p_{i}<q_{i}$, $i=1,2, \ldots$, из полуинтервала $[p, q[$. Положим $\alpha(0)=1$. Пусть для $i-1$ соответствующий выбор уже сделан. Зафиксируем $\alpha(i)>\alpha(i-1)$ и $p_{i}<q_{i}$ следующим образом.

Из (2) вытекает существование такого индекса $\alpha(i)>\alpha(i-1)$, что $\|z(s)\|=(r+1) \mu_{\alpha(i)}$ при некотором $s \in[p, q[$. Теперь опять же из (2) вытекает существование таких точек $p_{i}<q_{i}$ из $[p, q[$, что

$(*)(r+1) \mu_{\alpha(i)}<\|z(t)\|<(r+1+i) \mu_{\alpha(i)}$ при $p_{i}<t<q_{i}$ и $\left\|z\left(p_{i}\right)\right\|=(r+1) \mu_{\alpha(i)}$, $\left\|z\left(q_{i}\right)\right\|=(r+1+i) \mu_{\alpha(i)}$.

Пусть $A$ обозначает александровскую компактификацию пространства $\mathbb{R}^{n}$ (она получается из $\mathbb{R}^{n}$ добавлением бесконечно удаленной точки $a_{\infty}$ и гомеоморфна сфере $\left.S^{n}\right)$.

Пространство $\exp ([p, q] \times A)$ замкнутых подмножеств компакта $[p, q] \times A$ (в метрике Хаусдорфа) компактно, поэтому выбор в (*) мы можем сделать так, что дополнительно выполнено условие

$(* *)$ последовательность компактов $\operatorname{Gr}\left(\widetilde{\psi}_{\lambda_{\alpha(i)}}\left(\left.z\right|_{\left[p_{i}, q_{i}\right]}\right)\right)$ сходится в пространстве $\exp ([p, q] \times A)$ к некоторому множеству $H$.

Компакты в последовательности (**) связны, поэтому предельньй компакт $H$ также связен [4, теорема IV.8.8].

Так как $\left\|z\left(p_{i}\right)\right\|,\left\|z\left(q_{i}\right)\right\| \rightarrow \infty$, то из непрерьвности функции $z$ следует, что $p_{i}, q_{i} \rightarrow q$, и поэтому $H \subseteq\{q\} \times A$.

Покажем, что множество $H_{1}=\{u: u \in A,\{q, u\} \in H\}$ неограничено. Для любого $r_{1}>r+1$ найдется такой индекс $i_{0}=1,2, \ldots$, что $r_{1}<r+1+i_{0}$. При $i \geqslant i_{0}$ значения функции $\widetilde{\psi}_{\lambda_{\alpha(i)}}\left(\left.z\right|_{\left[p_{i}, q_{i}\right]}\right)$ меняются от $r+1$ до $r+1+i_{0}$, и поэтому ее график пересекается с множеством $\left\{(t, u): t \in[p, q],\|u\|=r_{1}\right\}$. Переходя к предельному множеству $H$ получаем

$$
H \cap\left\{(t, u): t \in[p, q],\|u\|=r_{1}\right\} \neq \varnothing,
$$

что и дает требуемое.

Компоненты связности множества $H_{1} \backslash\left\{a_{\infty}\right\}$ неограничены - это прямое следствие леммы 1.1.

Сопоставляя это с условием 4), получаем, что $H \cap V \neq \varnothing$ для некоторого $V \in \gamma$.

Найдутся точка $x=\left(q, x_{1}\right) \in H_{1} \cap V$ и число $\left.\varepsilon \in\right] 0,1\left[\right.$, для которьх $\left[O_{\varepsilon} x\right] \subseteq V$. Начиная с некоторого $i=i_{1}>\left\|x_{1}\right\|+1$, мы можем так отметить точки $s_{i} \in\left[p_{i}, q_{i}\right]$, что $\lambda_{\alpha(i)} z\left(s_{i}\right) \in O_{\varepsilon / 2} x$ и $\left(s_{i}, \lambda_{\alpha(i)} z\left(s_{i}\right)\right) \rightarrow x$ при $i \rightarrow \infty$.

При этом $\lambda_{\alpha(i)}\left\|z\left(q_{i}\right)\right\| \geqslant r+1+i \geqslant\|x\|+1+\varepsilon$, и поэтому при некотором $\left.\left.t_{i} \in\right] s_{i}, q_{i}\right]$

$$
\left(t_{i}, \lambda_{\alpha(i)} z\left(t_{i}\right)\right) \in \partial O_{\varepsilon} x, \quad \operatorname{Gr}\left(\left.\lambda_{\alpha(i)} z\right|_{] s_{i}, t_{i}[}\right) \subseteq O_{\varepsilon} x
$$

Последовательность функций $y_{i}=\left.\lambda_{\alpha(i)} z\right|_{]} s_{i}, t_{i}$ [ не является равностепенно непрерывной, так как $s_{i}, t_{i} \rightarrow q$ (поэтому $t_{i}-s_{i} \rightarrow 0$ ) и одновременно $\left\|y\left(s_{i}\right)-y\left(t_{i}\right)\right\| \rightarrow \varepsilon$. 
Графики функций $y_{i}$ лежат в компакте $\left[O_{\varepsilon} x\right] \subseteq V$, что вместе с предыдущим замечанием противоречит условию 3 ).

Сделанное предположение привело нас к противоречию. Теорема доказана.

В силу [4, лемма V.6.1] из теоремы 1.1 вытекает

СлЕДСТВИЕ. Пусть $\left.r \geqslant 0, V_{r}=\left\{u: u \in \mathbb{R}^{n},\|u\|>r\right\}, U_{r}=\right] a, b\left[\times V_{r}\right.$, $U=] a, b\left[\times \mathbb{R}^{n}\right.$ и $Z \in R_{\text {се }}(U)$. Пусть выполнены условия 2)-4) теоремы 1.1. Тогда если область определения функиии $z \in Z^{\mp}$ пересекается с отрезком $\left[t_{0}, t_{1}\right]$, то $\left[t_{0}, t_{1}\right] \subseteq \pi(z)$.

Прямым следствием теоремы 1.1 является также

ТЕОрема 1.2. Пусть выполнены условия 1) и 2) теоремы $1.1, Z_{\infty} \in R^{i}\left(U_{r}\right), \gamma-$ семейство открытых подмножеств области $U_{r}$, для любого әлемента $V$ которого $\left(\widetilde{\psi}_{i}(Z)\right)_{V} \rightarrow\left(Z_{\infty}\right)_{V}$ при $i \rightarrow \infty$. Пусть выполнено условие 4) теоремы 1.1. Тогда множество значений любой функиии из множества $M=\left\{z: z \in Z^{-} \cup Z^{+}, \pi(z) \subseteq\right.$ $\left.\left[t_{0}, t_{1}\right]\right\}$ является ограниченным.

В силу [4, лемма V.6.1] из теоремы 1.2 вытекает

СлЕДСТВИЕ. Пусть $\left.r \geqslant 0, V_{r}=\left\{u: u \in \mathbb{R}^{n},\|u\|>r\right\}, U_{r}=\right] a, b\left[\times V_{r}\right.$, $U=] a, b\left[\times \mathbb{R}^{n}\right.$ u $Z \in R_{\text {се }}(U)$. Пусть выполнено условие 2$)$ теоремы $1.1, Z_{\infty} \in R^{i}\left(U_{r}\right)$, $\gamma$ - семейство открытых подмножеств области $U_{r}$, для любого әлемента $V$ которого $\left(\widetilde{\psi}_{i}(Z)\right)_{V} \rightarrow\left(Z_{\infty}\right)_{V}$ при $i \rightarrow \infty$. Пусть выполнено условие 4) теоремы 1.1. Тогда если область определения функиии $z \in Z^{\mp}$ пересекается с отрезком $\left[t_{0}, t_{1}\right]$, $m o\left[t_{0}, t_{1}\right] \subseteq \pi(z)$.

В других ситуациях при использовании теоремы 1.1 и ее следствия для проверки выполнения условия 3) полезно простое

УТВЕРЖДЕНИЕ 1.1. Пусть $U$ - открытое подмножество пространства $\mathbb{R} \times \mathbb{R}^{n}$, $X$ - метрическое пространство, $\varphi_{0}: U \rightarrow X$ - непрерывное отображение, $\alpha=$ $\left\{Z_{i}: i=1,2, \ldots\right\} \subseteq R^{i}(U)$, для любого компакта $K \subseteq U$ и любой последовательности функиий $z_{j} \in\left(Z_{i_{j}}\right)_{K}, i_{1}<i_{2}<i_{3}<\cdots$, последовательность функиий $\varphi_{0}\left(t, z_{j}(t)\right), j=1,2, \ldots, t \in \pi\left(z_{j}\right)$, равностепенно непрерывна, $\gamma$ - семейство открытых подмнохеств мнохества $U$, для любого әлемента $V$ которого $\left\{\left(Z_{i}\right)_{V}: i=1,2, \ldots\right\} \in s(V), E=U \backslash \bigcup \gamma u$ для любых $t \in \mathbb{R} u x \in X$ мнохество $\left\{y:(t, y) \in E, \varphi_{0}(t, y)=x\right\}$ не содержит связных неодноточечных подмножсеств. Тогда $\alpha \in s(U)$.

ПримеР. Покажем, что максимально продолженные решения системы

$$
\left\{\begin{array}{l}
x^{\prime}=x+y+x^{100} e^{-y^{2}} \\
y^{\prime}=-x+y-x^{100} e^{-y^{2}}
\end{array}\right.
$$

определены на всей прямой $\mathbb{R}$. (Похожее на систему (3) в плане оценки правой части мажорантами скалярное уравнение $x^{\prime}=x^{100}$ этим свойством не обладает: его решения имеют вид

$$
x=-\sqrt[99]{\frac{1}{99 t+C}}
$$


и благополучно уходят на бесконечность за конечное время.)

Замена переменных $\psi_{\lambda}(t, y)=(t, \lambda y)$ переводит систему $(3)$ в систему

$$
\left\{\begin{array}{l}
x^{\prime}=x+y+\lambda^{-99} x^{100} e^{-(y / \lambda)^{2}}, \\
y^{\prime}=-x+y-\lambda^{-99} x^{100} e^{-(y / \lambda)^{2}} .
\end{array}\right.
$$

При $\lambda \rightarrow 0$ добавочные слагаемые в правой части системы (4) стремятся к нулю равномерно на любом компакте $K$ плоскости переменных $x, y$, который не пересекается с осью абсцисс $L$. Таким образом, условие 3 ) вьполнено для семейства $\gamma$ всех открытых ограниченных множеств вида $G \times W$, где $W$ - подмножество плоскости переменных $x, y$, не пересекающееся с осью абсцисс.

Пусть компактное подмножество $K$ плоскости переменных $x, y$ не содержит начала координат и $\alpha(x, y)=x+y$. Для любого решения $z(t)=(x(t), y(t))$ системы $(4)$, множество значений которого лежит в компакте $K$, в силу равенства $(\alpha(z(t)))^{\prime}=2 y^{\prime}(t)$ справедлива оценка

$$
\left|(\alpha(z(t)))^{\prime}\right| \leqslant M
$$

где $M=2 \sup \{\|u\|: u \in K\}$.

Для любого числа $l \in \mathbb{R}$ множество $\alpha^{-1}(l) \cap L$ состоит из одной точки. В силу сказанного по утверждению 1.1 множество $Z_{K}$ равностепенно непрерывно для любого компакта $K \subseteq \mathbb{R} \times \mathbb{R}^{2}$, проекция которого на плоскость переменных $x, y$ не содержит начала координат. Поэтому множество $Z_{V}$ равностепенно непрерьвно для любого открытого множества $V$ вида $G \times W$, где $W$ - подмножество плоскости, не содержащее начала координат.

Теперь для получения требуемого нам остается применить к системе (3) теорему 1.1.

Ранее результаты об оценке длины интервала существования решения получались, исходя из оценки скорости роста правой части. Предполагалось, что правая часть имеет не более чем линейный рост (см. например, [8, гл. 7]), или близкие условия (см. например, $[9, \S 5.1])$. При переходе к нашим топологическим структурам мы переходим от метрических оценок асимптотики правой части через неравенства (“линейный рост") к оценкам асимптотики топологического строения пространств решений. Наши оценки, в принципе, имеют в виду аналогичные связи, но являются более объемными. Нет необходимости пояснять, что множитель $x^{100}$ в последних слагаемых уводит нас в примере от предположения линейности роста.

\section{§2. Об ацикличности множества решений задачи Коши}

Предположим, что $U$ - открытое подмножество произведения $\mathbb{R} \times \mathbb{R}^{n},\left(a, y_{0}\right) \in U$, $b>a$.

Наша ближайшая цель - научиться доказывать ацикличность множеств $S_{Z}\left(y_{0} ; a, b\right)=$ $\left\{z: z \in Z, \pi(z)=[a, b], z(a)=y_{0}\right\}$, т.е. выполнение условия

$(h)$ в указанных вьше обозначениях

$$
H_{i}\left(S_{Z}\left(y_{0} ; a, b\right)\right)= \begin{cases}\mathbb{Q} & \text { при } i=0, \\ 0 & \text { при } i \neq 0\end{cases}
$$

для любой точки $\left(a, y_{0}\right) \in U$ и любого отрезка $[a, b] \subseteq \mathbb{R}$, удовлетворяющего условию $[a, b] \subseteq \pi(z)$ для любого решения задачи Коши $z \in Z^{\mp}, z(a)=y_{0}$. 
(Здесь и дальше имеются в виду рациональные гомологии, см. также [1].)

Начнем с предварительных рассмотрений.

УТВЕРЖДЕНИЕ 2.1. Пусть $U$ - открытое подмножество произведения $\mathbb{R} \times \mathbb{R}^{n}$, $Z \subseteq C_{s}(U), S_{Z}\left(y_{0} ; a, b\right)=\left\{z: z \in Z, \pi(z)=[a, b], z(a)=y_{0}\right\}$, последовательность пространств $\left\{Z_{i}: i=1,2, \ldots\right\}$ сходится $\kappa$ пространству $Z, K, K_{1}$ - компактные подмножества множества $U, K \subseteq\left\langle K_{1}\right\rangle$,

$$
\left\{z: z \in Z, \operatorname{Gr}(z) \subseteq K_{1},(\inf \pi(z), z(\inf \pi(z))) \in K,(\sup \pi(z), z(\sup \pi(z))) \in \partial K_{1}\right\}=\varnothing
$$

Тогда найдется такой индекс $i_{0}=1,2, \ldots$, что если

$$
\begin{aligned}
z \in \bigcup\left\{Z_{i}: i=\right. & \left.i_{0}, i_{0}+1, \ldots\right\}, \quad \pi(z) \subseteq[a, b], \quad \operatorname{Gr}(z) \subseteq K_{1} \\
& (\sup \pi(z), z(\sup \pi(z))) \in \partial K_{1}
\end{aligned}
$$

$m o \operatorname{Gr}(z) \cap K=\varnothing$.

ДокАЗАтЕльство. Допустим противное. Тогда для любого $j=1,2, \ldots$ найдется такая функция $z_{j} \in \bigcup\left\{Z_{i}: i=j, j+1, j+2, \ldots\right\}$, что $\pi\left(z_{j}\right) \subseteq[a, b], \operatorname{Gr}\left(z_{j}\right) \subseteq K_{1}$, $\left(\sup \pi\left(z_{j}\right), z_{j}\left(\sup \pi\left(z_{j}\right)\right)\right) \in \partial K_{1}$ и $\left(\inf \pi\left(z_{j}\right), z_{j}\left(\inf \pi\left(z_{j}\right)\right)\right) \in K$.

Для каждого $j=1,2, \ldots$ отметим такой индекс $i(j) \geqslant j$, для которого $z_{j} \in Z_{i(j)}$.

От последовательности $\left\{z_{j}: j=1,2, \ldots\right\}$ перейдем к подпоследовательности $\left\{z_{j_{k}}\right.$ : $k=1,2, \ldots\}$, для которой $i\left(j_{1}\right)<i\left(j_{2}\right)<i\left(j_{3}\right)<\cdots$. Графики всех этих функций лежат в компакте $K_{1}$, и поэтому сходимость $Z_{i} \rightarrow Z$ влечет возможность выбора из последовательности $\left\{z_{j_{k}}: k=1,2, \ldots\right\}$ подпоследовательности, сходящейся к некоторой функции $z_{0} \in Z_{K_{1}}$. Для нее в силу теорем III.5.1 и III.5.4 из [4]

$$
\begin{gathered}
\left(\inf \pi\left(z_{0}\right), z_{0}\left(\inf \pi\left(z_{0}\right)\right)\right) \in K, \\
\pi\left(z_{0}\right) \subseteq[a, b], \\
\left(\sup \pi\left(z_{0}\right), z_{0}\left(\sup \pi\left(z_{0}\right)\right)\right) \in \partial K_{1} .
\end{gathered}
$$

Но из условий (5) и (6) следует, что график $\operatorname{Gr}\left(z_{0}\right)$ функции $z_{0}$ лежит в компакте $K$, а это очевидным образом противоречит предположениям формулировки утверждения и (7). Мы получили требуемое. Утверждение доказано.

УТВЕРЖДЕНИЕ 2.2. Пусть $U$ - открытое подмножество произведения $\mathbb{R} \times \mathbb{R}^{n}$, $Z \in\left[R_{\text {сеи }}(U)\right]_{R_{c}(U)}$. Тогда пространство $Z$ удовлетворяет условию $(h)$.

ДокАЗАтЕЛЬСтво. Пусть $\left(a, y_{0}\right) \in U, b>a$. Пусть $[a, b] \subseteq \pi(z)$ для любого решения задачи Коши $z \in Z^{\mp}, z(a)=y_{0}$. Пусть $\Lambda=\left\{z: z \in C\left([a, b], \mathbb{R}^{n}\right), \operatorname{Gr}(z) \subseteq U\right\}$.

Мы получим требуемое, если покажем, что для любой окрестности $W$ множества $S=S_{Z}\left(y_{0} ; a, b\right)$ в пространстве $\Lambda$ существует гомотопия $F: S \times I \rightarrow W$, начинающаяся с тождественного отображения и заканчивающаяся отображением в точку. Сделаем это.

I. По теореме IV.8.7 из [4] множество $K=\bigcup\{\operatorname{Gr}(z): z \in S\}(\subseteq U)$ компактно.

По лемме II.4.2 из [3] найдется такое число $\delta_{1}>0$, что множество $K_{1}=\left[O_{\delta_{1}} K\right]-$ компакт, лежащий в $U$. 
II. Зафиксируем последовательность $\left\{Z_{i}: i=1,2, \ldots\right\} \subseteq R_{c e u}(U)$, сходящуюся в пространстве $R_{c}(U)$ к пространству $Z$.

III. По утверж дению 2.1 найдется такой индекс $i_{0}=1,2, \ldots$, что если $z \in \bigcup\left\{Z_{i}: i=i_{0}\right.$, $\left.i_{0}+1, \ldots\right\}, \pi(z) \subseteq[a, b], \operatorname{Gr}(z) \subseteq K_{1},(\sup \pi(z), z(\sup \pi(z))) \in \partial K_{1}$, то $\operatorname{Gr}(z) \cap K=\varnothing$.

$I V$. Пусть для $a \leqslant s \leqslant b$ и $p=1,2, \ldots$ символ $G(s, p)$ обозначает множество всех функций $z \in C_{s}(U)$, удовлетворяющих условиям:

$$
\left.z\right|_{]-\infty, s] \cap \pi(z)} \in Z,\left.\quad z\right|_{[s, \infty[\cap \pi(z)} \in Z_{i}
$$

При введении в рассмотрение множества $G(s, p)$ мы использовали операцию подклеивания пространств, изученную в [4, § VIII.7].

Пусть $H(p)=\bigcup\{G(s, p): a \leqslant s \leqslant b\}$ и $H_{1}(p)=\left\{z: z \in H(p), \pi(z)=[a, b], z(a)=y_{0}\right\}$.

Из III вытекает, что при $p \geqslant i_{0}$ графики функций из $H_{1}(p)$ лежат в множестве $O_{\delta} K$.

Отсюда из теоремы VIII.7.1 и замечаний $§$ VIII.7 в [4] вытекает компактность множества $H_{1}(p)$ при $p \geqslant i_{0}$.

$V$. Покажем, что при $p \rightarrow \infty$ компакты $H_{1}(p)$ сходятся к компакту $S$ в смысле вьполнения условия

$(*)$ из любой последовательности точек $x_{j} \in H_{1}\left(p_{j}\right), p_{1}<p_{2}<\cdots$, можно выбрать подпоследовательность, сходящуюся к некоторой точке множества $S$.

Допустим противное. Тогда найдутся последовательности индексов $\{p(l): l=1$, $2, \ldots\}$ и функций $z_{l} \in H_{1}(p(l))$ таких, что из последовательности функций $\left\{z_{l}: l=1\right.$, $2, \ldots\}$ нельзя выбрать подпоследовательности, сходящейся к некоторому элементу множества $S$.

Но $z_{l} \in G(s(l), p(l))$ при некотором $s(l) \in[a, b]$. Так как числа $s(l)$ меняются в отрезке $[a, b]$, мы можем перейти к такому бесконечному множеству $A \subseteq \mathbb{N}$, что существует предел

$$
s=\lim _{\substack{l \in A \\ l \rightarrow \infty}} s(l) .
$$

В силу условия $Z_{i} \rightarrow Z$ и (*) последовательность функций $\left\{\left.z_{l}\right|_{[s(l), b]}: l=1,2, \ldots\right\}$ сходится к некоторой функции $\varphi_{1} \in Z$, заданной на отрезке $[s, b]$.

В силу принадлежности $Z \in R_{c}(U)$ последовательность функций $\left\{\left.z_{l}\right|_{[a, s(l)]}: l=1\right.$, $2, \ldots\}$ сходится к некоторой функции $\varphi \in Z$, заданной на отрезке $[a, s]$.

Из теоремы III.5.4 в [4] вытекает, что $\varphi(s)=\varphi_{1}(s)$ и поэтому функция

$$
z(t)= \begin{cases}\varphi(t) & \text { при } t \in[a, s[, \\ \varphi_{1}(t) & \text { при } t \in[s, b[\end{cases}
$$

непрерьвна. Из теоремы III.5.5 в [4] вытекает сходимость $z_{l} \rightarrow z$ при $l \in A$ и $l \rightarrow \infty$, что противоречит первоначальному выбору последовательности $\left\{z_{l}: l=1,2, \ldots\right\}$.

VI. Из п. III и IV нашего доказательства и леммы III.5.1 в [4] вытекает существование такого индекса $i_{1} \geqslant i_{0}$, что $H_{1}(p) \subseteq W$ при $p \geqslant i_{1}$.

Так как при этом $S \subseteq H_{1}(p)$, то нам остается показать, что компакт $H_{1}(p)$ стягиваем.

Пусть $z \in H_{1}(p)$ и $s \in[a, b]$. Обозначим через $\xi_{s}(z)$ ту единственную функцию $\zeta \in Z_{p}^{+}$, для которой $\inf \pi(\zeta)=s$ и $\zeta(s)=z(s)$. 
Обозначим через $\sigma_{s}(z)$ функцию

$$
z_{1}(t)= \begin{cases}z(t) & \text { при } t \in[a, s], \\ \xi_{s}(t) & \text { при } t \in[s, b] .\end{cases}
$$

Функция $\sigma_{s}(z)$ принадлежит $H_{1}(p)$. Таким образом, мы можем определить функцию двух переменных $F(z, s)=\sigma_{s}(z)$.

Ее непрерьвность по совокупности аргументов следует из теоремы VIII.7.1 в [3]. Таким образом, мы имеем гомотопию $F: H_{1}(p) \times[a, b] \rightarrow H_{1}(p)$. При этом $F(z, b) \equiv z$ и $F(z, a)$ есть единственное решение задачи Коши $y \in Z_{p}, y(a)=y_{0}$ на отрезке $[a, b]$. То есть гомотопия $F$ стягивает компакт $H_{1}(p)$ в точку. Утверждение доказано.

Сделаем несколько замечаний по поводу условия $Z \in\left[R_{c e u}(U)\right]_{R_{c}(U)}$. Если для пространства решений написанного без особых ухищрений уравнения выполнены условия $(c)$ и $(e)$, то скорее всего будет вьполнено и условие $Z \in\left[R_{c e u}(U)\right]$. По крайней мере, примеры ситуаций, когда это не так, нужно строить с дополнительными усилиями. Более того, наиболее естественньй способ доказательства выполнения условия $(e)$ (теоремы существования решения задачи Коши) опирается на построение аппроксимации, соответствующей принадлежности $Z \in\left[R_{c e u}(U)\right]$ (см. [2, теорема 3.1]). Так что ацикличность множества решений задачи Коши доказьвается на этом пути в силу утверждения 2.2 одновременно с теоремой сушествования решения задачи Коши. Для уравнений в предположениях Каратеодори и для соответствующих дифференциальных включений такая аппроксимация известна (см. [1]). Наличие общего утверждения 2.2 на эту тему позволяет исследовать уравнения с особенностями. Как это делается, мы увидим в примере в конце статьи, где утверждение 2.2 используется для доказательства существования периодического решения.

Заметим теперь, что вьполнение условия $(h$ ) (и это существенно при исследовании уравнений с особенностями и включений) носит локальный характер, а именно справедливо

УТВЕРЖДЕНИЕ 2.3. Пусть $U-$ открытое подмножсество произведения $\mathbb{R} \times \mathbb{R}^{n}$, $Z \in R_{\text {се }}(U), \gamma$-открытое покрытие области $U$, для любого әлемента $V$ которого $Z_{V} \in R_{h}(V)$. Тогда $Z \in R_{h}(U)$.

(Здесь в соответствии с общей схемой обозначений [2]-[4] запись $Z \in R_{h}(U)$ означает выполнение условия $(h)$.)

ДокАЗАтЕЛЬСтво. Пусть $\left(a, y_{0}\right) \in U, b>a$. Пусть $[a, b] \subseteq \pi(z)$ для любого решения задачи Коши $z \in Z^{\mp}, z(a)=y_{0}$.

Множество $M$ точек $t$ отрезка $[a, b]$, удовлетворяющих условию

$$
H_{i}\left(S_{Z}\left(y_{0} ; a, s\right)\right)= \begin{cases}\mathbb{Q} & \text { при } i=0, \\ 0 & \text { при } i \neq 0\end{cases}
$$

для всех $s \in[a, t]$, непусто: оно содержит по крайней мере точку $a$.

Наша цель будет достигнута, если мы покажем, что из $c \in[M]$ следует, что $c \in\langle M\rangle$ (замыкание $[M]$ и внутренность $\langle M\rangle$ берутся относительно отрезка $[a, b]$ ).

В силу выбора $b$ и $c$ из леммы VII.1.1 в [4] вытекает, что множество $H=\{z(c): z \in$ $\left.S_{Z}\left(y_{0} ; a, t\right)\right\}$ компактно, поэтому из семейства $\gamma$ мы можем выбрать конечное число элементов $V_{1}, \ldots, V_{p}$, в совокупности покрывающих множество $H: H \subseteq V_{1} \cup \cdots \cup V_{p}$. 
Функции $\varphi_{i}(x)=\rho\left(x, \mathbb{R}^{n+1} \backslash V_{i}\right), i=1, \ldots, p, \varphi(x)=\max \left\{\varphi_{1}(x), \ldots, \varphi_{p}(x)\right\}$ непрерывны и функция $\left.\varphi\right|_{H}$ строго положительна. В силу компактности множества $H$ число $\varepsilon=\frac{1}{2} \inf \varphi(H)$ положительно. В силу определения $\varepsilon$ компактные подмножества

$$
H_{i}=\left\{x: x \in \mathbb{R}^{n+1}, \varphi_{i}(x) \geqslant 2 \varepsilon\right\}
$$

пространства $\mathbb{R}^{n+1}$ покрывают компакт $H: H=H_{1} \cup \cdots \cup H_{p}$ и $H_{i} \subseteq V_{i}$ (при любом $i=1, \ldots, p)$.

Замыкание множества $G_{i}=O_{\varepsilon}\left(H_{i} \cap H\right)$ компактно и лежит в $V_{i}:\left[G_{i}\right] \subseteq V_{i}$.

Применив лемму V.6.3 из [4] к компактам $\left[G_{i}\right]$ и областям $V_{i}$, получаем существование такого $\delta>0$, что если

$$
z \in Z^{\mp}, \quad \operatorname{Gr}(z) \cap G_{i} \ni(s, z(s)),
$$

то $[s-\delta, s+\delta] \subseteq \pi(z)$ и $\operatorname{Gr}\left(\left.z\right|_{[s-\delta, s+\delta]}\right) \subseteq V_{i}$.

Далее, при некотором $\left.\left.\delta_{1} \in\right] 0, \delta\right]$ множество $\left\{(t, z(t)): z \in S_{Z}\left(y_{0} ; a, t\right)\right\}$ лежит в множестве $G=G_{1} \cup \cdots \cup G_{p}$ при любом $\left.t \in\right] c-\delta_{1}, c+\delta_{1}[$ (это следует, например, из компактности множества $S_{Z}\left(y_{0} ; a, b\right)$ и следствия теоремы III.5.4 в [4]).

Возьмем произвольно $\left.c_{1} \in\right] c-\delta_{1} / 2, c\left[\right.$ и $\left.c_{2} \in\right] c, c+\delta_{1} / 2[$ и рассмотрим отображение

$$
\Phi: S_{Z}\left(y_{0} ; a, c_{2}\right) \rightarrow S_{Z}\left(y_{0} ; a, c_{1}\right)
$$

заданное формулой $\Phi(z)=\left.z\right|_{\left[a, c_{2}\right]}$.

По выбору точки $c_{2}$ множество $S_{Z}\left(y_{0} ; a, c_{1}\right)$ ациклично. Возьмем произвольно $z_{1} \in$ $S_{Z}\left(y_{0} ; a, c_{1}\right)$. Точка $z_{1}\left(c_{1}\right)$ принадлежит одному из множеств $G_{i}, i=1, \ldots, p$. В силу $(8)$ и оценки $c_{2}-c_{1} \leqslant \delta_{1}<\delta$ графики всех функций из множества

$$
\left\{\left.z\right|_{\left[c_{1}, c_{2}\right]}: z \in \Phi^{-1}\left(z_{1}\right)\right\}=S_{Z}\left(y_{0} ; c_{1}, c_{2}\right)
$$

лежат в $V_{i}$, и в силу принадлежности $V_{i} \in \gamma$ множество $\Phi^{-1}\left(z_{1}\right)$ ациклично. По теореме 2.1 из [1] из сказанного вытекает ацикличность множества $S_{Z}\left(y_{0} ; a, c_{2}\right)$ и затем включение $\left[a, c+\delta_{1}[\subseteq M\right.$. Утверждение доказано.

\section{§3. Степени отображений}

В этом параграфе мы внесем в схему понятий из [1] необходимые нам изменения. Рассмотрим следующую ситуацию.

А. $U$ - открытое подмножество произведения $\mathbb{R} \times \mathbb{R}^{n},\left[t_{0}, t_{1}\right] \subseteq \mathbb{R},-\infty<c \leqslant d<\infty$, $S^{n-1}=\left\{u: u \in \mathbb{R}^{n},\|u\|=1\right\}$ (т.е. единичная сфера пространства $\mathbb{R}^{n}$ ), $X_{0}=[c, d] \times S^{n-1}$, для $s \in[c, d] Z_{s} \in R_{c e}(U)$ и отображение $\psi:[c, d] \rightarrow R_{c}(U)$, заданное формулой $\psi(s)=Z_{s}$, непрерывно, функции $\theta_{1}, \theta_{2}:[c, d] \rightarrow\left[t_{0}, t_{1}\right]$ непрерывны, $\theta_{1}(s)<\theta_{2}(s)$ при любом $s \in[c, d], \varphi: X_{0} \rightarrow \mathbb{R}^{n}$ - непрерывное отображение и $\left\{\theta_{1}(s)\right\} \times \varphi\left(\{s\} \times S^{n-1}\right) \subseteq U$ при любом $s \in[c, d]$, при любом $s \in[c, d]$ любое решение задачи Коши $z \in Z_{s}, z\left(\theta_{1}(s)\right) \in$ $\varphi\left(\{s\} \times S^{n-1}\right)$ продолжается на отрезок $\left[\theta_{1}(s), \theta_{2}(s)\right]$ и $z\left(\theta_{2}(s)\right) \neq z\left(\theta_{1}(s)\right)$, при любых $s \in[c, d]$ и $u \in S^{n-1}$ множество $\left\{z: z \in Z_{s}, \pi(z)=\left[\theta_{1}(s), \theta_{2}(s)\right], z\left(\theta_{1}(s)\right)=\varphi(s, u)\right\}$ ациклично.

(Два предыдущих параграфа были посвящены обсуждению последних двух условий из А.) 
По лемме VII.1.1 из [4] при любом $s \in[c, d]$ множество

$$
X(s)=\left\{z: z \in Z_{s}, \pi(z)=\left[\theta_{1}(s), \theta_{2}(s)\right], z\left(\theta_{1}(s)\right) \in \varphi\left(\{s\} \times S^{n-1}\right)\right\}
$$

компактно. Из леммы VI.7.2 и теоремы II.5.1 в [4] следует полунепрерьвность сверху отображения, сопоставляющего точке $s \in[c, d]$ компактное подмножество $X(s)$ пространства $C_{s}(U)$. Отсюда и из теоремы I.7.8 в [4] вытекает компактность множества $X_{1}=\bigcup\{X(s): s \in[c, d]\}$.

Множество

$$
X=\left\{((s, u), z):(s, u) \in X_{0}, z \in X(s), z\left(\theta_{1}(s)\right)=\varphi(s, u)\right\}
$$

является замкнутым подмножеством компакта $X_{0} \times X_{1}$ и потому компактно.

Пусть $p: X \rightarrow X_{0}$ - отображение, порожденное проектированием $X_{0} \times X_{1} \rightarrow X_{0}$. Последнее из условий означает ацикличность прообразов точек при отображении $p$.

В силу теоремы 1.2 из [1] гомоморфизмы $p_{*}: H_{i}(X) \rightarrow H_{i}\left(X_{0}\right)$, порожденные непрерьвньг отображением $p$, являются изоморфизмами. Пусть $p_{1}: H_{i}\left(X_{0}\right) \rightarrow H_{i}(X)$ - обратные изоморфизмы. Условие

при любом $s \in[c, d]$ любое решение задачи Коши $z \in Z_{s}, z\left(\theta_{1}(s)\right) \in \varphi\left(\{s\} \times S^{n-1}\right)$

продолжается на отрезок $\left[\theta_{1}(s), \theta_{2}(s)\right]$

означает, что при $s \in[c, d]$ и $z \in X(s)$ точка $q(s, u)=z\left(\theta_{2}(s)\right)-z\left(\theta_{1}(s)\right)$ лежит в множестве $Y=\mathbb{R}^{n} \backslash\{\overline{0}\}$.

Таким образом, отображение $q: X \rightarrow Y$ порождает гомоморфизмы $q_{*}: H_{i}(X) \rightarrow H_{i}(Y)$.

Группы $H_{n-1}\left(X_{0}\right)$ и $H_{n-1}(Y)$ изоморфны группе $\mathbb{Q}=H_{n-1}\left(S^{n-1}\right)$. Изоморфизм $H_{n-1}\left(X_{0}\right) \rightarrow H_{n-1}\left(S^{n-1}\right)$ порождается проектированием

$$
X_{0}=[c, d] \times S^{n-1} \rightarrow S^{n-1} .
$$

Изоморфизм

$$
H_{n-1}(Y) \rightarrow H_{n-1}\left(S^{n-1}\right)
$$

порождается непрерывным отображением, заданным соответствием

$$
u \mapsto \frac{u}{\|u\|} .
$$

Обозначим через е элементы этих груп, являющиеся образами единищы при указанных изоморфизмах. Образ элемента $е$ групшы $H_{n-1}\left(X_{0}\right)$ при гомоморфизме $q_{*} p_{1}$ имеет вид $k e$. Число $k$ будем назьвать индексом семейства пространств $Z_{s}$ относительно тройки $\left(\varphi, \theta_{1}, \theta_{2}\right)$ и обозначать $k\left(Z_{s}, \varphi, \theta_{1}, \theta_{2}\right)$.

Рассмотрим частньй случай $c=d$. Здесь можно опустить упоминание об отрезке $[c, d]$ (он вырождается в одноточечное множество $\{c\}$ ), об отображениях $\theta_{1}, \theta_{2}$ и о полуинтервалах $\left[t_{0}, \theta_{1}(c)[\right.$ и $\left.] \theta_{2}(c), t_{1}\right]$, точки которых в этой ситуации в дальнейших рассмотрениях не участвуют, положив $\theta_{1}(c)=t_{0}$ и $\theta_{2}(c)=t_{1}$. Семейство пространств $\left\{Z_{s}: s \in[c, d]\right\}$ оказьвается состоящим из одного пространства $Z=Z_{c}$. В этой ситуации число $k\left(Z_{s}, \varphi, \theta_{1}, \theta_{2}\right)$ будем называть индексом пространства $Z$ относительно отображения $\varphi$ на отрезке $\left[t_{0}, t_{1}\right]$ и обозначать $k_{1}\left(Z, \varphi, t_{0}, t_{1}\right)$.

УТВЕРЖДЕНИЕ 3.1. В предположсения $x$ A

$$
k_{1}\left(Z_{c}, \varphi_{c}, \theta_{1}(c), \theta_{2}(c)\right)=k_{1}\left(Z_{d}, \varphi_{d}, \theta_{1}(d), \theta_{2}(d)\right),
$$

әде $\varphi_{c}$ - отобрахсние $S^{n-1} \rightarrow X_{0}$, заданное формулой $\varphi_{c}(u)=\varphi(c, u), \varphi_{d}-$ отобрахсение $S^{n-1} \rightarrow X_{0}$, заданное формулой $\varphi_{d}(u)=\varphi(d, u)$. 
ДокАЗАТЕЛЬСтво. Вложения $i_{c}, i_{d}: S^{n-1} \rightarrow X_{0}$, заданные формулами $i_{c}(u)=(c, u)$ и $i_{d}(u)=(d, u)$, индуцируют одни и те же изоморфизмы групп гомологий $H_{i}\left(S^{n-1}\right) \rightarrow$ $H_{i}\left(X_{0}\right)$, что и дает требуемое.

УТВЕРЖДЕНИЕ 3.2. Пусть $U$ - открытое подмножсество произведения $\mathbb{R} \times \mathbb{R}^{n}$, $\left[t_{0}, t_{1}\right] \subseteq \mathbb{R}, Z \in R_{c e h}(U),\left\{Y_{i}: i=1,2, \ldots\right\} \subseteq R_{c e h}(U) u Y_{i} \rightarrow Z n p u i \rightarrow \infty$, $\varphi_{0}: S^{n-1} \rightarrow \mathbb{R}$ - непрерывное отображсение $u\left\{t_{0}\right\} \times \varphi_{0}\left(S^{n-1}\right) \subseteq U$, любое решение задачи Коши $z \in Z, z\left(t_{0}\right) \in \varphi_{0}\left(S^{n-1}\right)$ продолжсается на отрезок $\left[t_{0}, t_{1}\right]$ и при этом $z\left(t_{1}\right) \neq z\left(t_{0}\right)$.

Тогда найдется такой индекс $i_{0}=1,2, \ldots$, что для любого $i=i_{0}, i_{0}+1, \ldots$

1) любое решение задачи Коши

$$
z \in Y_{i}, \quad z\left(t_{0}\right) \in \varphi_{0}\left(S^{n-1}\right)
$$

продолжается на отрезок $\left[t_{0}, t_{1}\right]$ и при этом $z\left(t_{1}\right) \neq z\left(t_{0}\right)$;

2) $k_{1}\left(Z, \varphi_{0}, t_{0}, t_{1}\right)=k_{1}\left(Y_{i}, \varphi_{0}, t_{0}, t_{1}\right)$.

ДокАЗАТЕЛЬСТво. I. По лемме VII.1.1 в [4] множество

$$
A=\left\{z: z \in Z, \pi(z)=\left[t_{0}, t_{1}\right], z\left(t_{0}\right) \in \varphi_{0}\left(S^{n-1}\right)\right\}
$$

компактно. Отсюда следует компактность множества $K=\bigcup\{\operatorname{Gr}(z): z \in A\}$.

Пусть число $\delta>0$ таково, что компакт $K_{1}=\left[O_{\delta} K\right]$ лежит в $U$. По утверждению 2.1 найдется такой индекс $i_{1}=1,2, \ldots$, что если

$$
\begin{aligned}
z \in \bigcup\left\{Y_{i}: i=\right. & \left.i_{1}, i_{1}+1, \ldots\right\}, \quad \pi(z) \subseteq[a, b], \quad \operatorname{Gr}(z) \subseteq K_{1}, \\
& (\sup \pi(z), z(\sup \pi(z))) \\
& \in \partial K_{1},
\end{aligned}
$$

то $\operatorname{Gr}(z) \cap K=\varnothing$.

II. Покажем, что найдется такой индекс $i_{0}=i_{1}, i_{1}+1, \ldots$, что если

$$
\begin{gathered}
z \in \bigcup\left\{Y_{i}: i=i_{0}, i_{0}+1, \ldots\right\}, \quad \pi(z) \subseteq\left[t_{0}, t_{1}\right], t_{1}=\sup \pi(z), s_{1}=\inf \pi(z), \\
y \in A, \quad y\left(s_{1}\right)=z\left(s_{1}\right),
\end{gathered}
$$

то $y\left(t_{0}\right) \neq z\left(t_{1}\right)$.

Допустим, что это не так. Тогда мы можем так выбрать последовательности индексов $i_{1}<j_{1}<j_{2}<\cdots$ и функций $z_{m} \in\left(Z_{j_{m}}\right)_{K_{1}}, y_{m} \in A$, что

$$
\pi(z) \subseteq\left[t_{0}, t_{1}\right], \quad t_{1}=\sup \pi(z), \quad y_{m}\left(\inf \pi\left(z_{m}\right)\right)=z_{m}\left(\inf \pi\left(z_{m}\right)\right)
$$

и $y\left(t_{0}\right)=z\left(t_{1}\right)$.

В силу компактности множества $A$ и сходимости $Y_{i} \rightarrow Z$ мы можем предположить дополнительно, что последовательность функций $\left\{y_{m}: m=1,2, \ldots\right\}$ сходится к некоторой функции $\widetilde{y} \in A$, а последовательность функций $\left\{z_{m}: m=1,2, \ldots\right\}$ сходится к некоторой функции $\widetilde{z} \in Z$. При этом

$$
\widetilde{y}(\inf \pi(\widetilde{z}))=\lim _{m \rightarrow \infty} y_{m}\left(\inf \pi\left(z_{m}\right)\right)=\lim _{m \rightarrow \infty} z_{m}\left(\inf \pi\left(z_{m}\right)\right)=\widetilde{z}(\inf \pi(\widetilde{z}))
$$


и поэтому функция

$$
\bar{y}(t)= \begin{cases}\widetilde{y}(t) & \text { при } t \in\left[t_{0}, \inf \pi(\widetilde{z})\right] \\ \widetilde{z}(\inf \pi(\widetilde{z})) & \text { при } t \in\left[\inf \pi(\widetilde{z}), t_{1}\right]\end{cases}
$$

принадлежит множеству $A$. Но

$$
\bar{y}\left(t_{0}\right)=\lim _{m \rightarrow \infty} y_{m}\left(t_{0}\right)=\lim _{m \rightarrow \infty} z_{m}\left(t_{1}\right)=\widetilde{z}\left(t_{1}\right)=\bar{y}\left(t_{1}\right)
$$

что противоречит предположениям формулировки утверждения.

III. Для $s \in\left[t_{0}, t_{1}\right]$ обозначим через $Z_{s}$ пространство функций $z \in C_{s}(U)$, удовлетворяющих условию

$$
\left.z\right|_{]-\infty, s] \cap \pi(z)} \in Z,\left.\quad z\right|_{[s, \infty[\cap \pi(z)} \in Y_{i_{0}}
$$

Полагаем $c=t_{0}, d=t_{1}, \theta_{1} \equiv t_{0}, \theta_{2} \equiv t_{1}, \varphi(s, u)=\varphi_{0}(u)$.

Выполнение условия $Z_{s} \in R_{c e}(U)$ следует из теоремы VIII.7.1 в [4].

Для доказательства последнего из условий А рассматриваем отображение

$\Psi:\left\{z: z \in Z_{s}, \pi(z)=\left[t_{0}, t_{1}\right], z\left(t_{0}\right)=\varphi_{0}(u)\right\} \rightarrow\left\{z: z \in Z_{s}, \pi(z)=\left[t_{0}, s\right], z\left(t_{0}\right)=\varphi_{0}(u)\right\}$

заданное формулой $\Psi(z)=\left.z\right|_{\left[t_{0}, s\right]}$.

Так как при этом ацикличны и множество значений отображения $\Psi$, и прообразы точек, то в силу теоремы 1.2 из [1] это дает требуемое.

Таким образом, мы находимся в предположениях утверждения 3.1 , что и дает требуемое. Утверждение доказано.

СлеДСтвиЕ. Пусть $U$ - открытое подмножество $\mathbb{R} \times \mathbb{R}^{n},\left[t_{0}, t_{1}\right] \subseteq \mathbb{R}, Z \in$ $R_{\text {сеһ }}(U), \varphi_{0}: S^{n-1} \rightarrow \mathbb{R}$ - непрерывное отображсение $u\left\{t_{0}\right\} \times \varphi_{0}\left(S^{n-1}\right) \subseteq U$, любое решение задачи Коши $z \in Z, z\left(t_{0}\right) \in \varphi_{0}\left(S^{n-1}\right)$ продолжается на отрезок $\left[t_{0}, t_{1}\right] u$ при этом $z\left(t_{1}\right) \neq z\left(t_{0}\right), Y \in R_{c e h}(U) u Y \subseteq Z$. Tогда $k_{1}\left(Z, \varphi_{0}, t_{0}, t_{1}\right)=k_{1}\left(Y, \varphi_{0}, t_{0}, t_{1}\right)$.

Для получения следствия достаточно рассмотреть последовательность $Y_{i} \equiv Y$.

\section{§4. О существовании периодических решений}

Рассмотрим следующую ситуацию.

A. $\left.-\infty<a<t_{0}<t_{1}<b<\infty, U=\right] a, b\left[\times \mathbb{R}^{n}, K_{r}=\left\{u: u \in \mathbb{R}^{n},\|u\| \leqslant r\right\}\right.$, $S_{r}=\left\{u: u \in \mathbb{R}^{n},\|u\|=r\right\}$, отображение $\varphi_{r}: S^{n-1} \rightarrow S_{r}$ задано формулой $\varphi_{r}(u)=r u$, любое решение задачи Коши $z \in Z, z\left(t_{0}\right) \in K_{r}$ продолжается на отрезок $\left[t_{0}, t_{1}\right]$.

В предположениях $\left.-\infty<a<t_{0}<t_{1}<b<\infty, U=\right] a, b\left[\times \mathbb{R}^{n}, Z \in R_{c e h}(U)\right.$ либо

$(*)$ найдется такая функция $z \in Z$, что $\pi(z)=\left[t_{0}, t_{1}\right]$ и $z\left(t_{0}\right)=z\left(t_{1}\right) \in S_{r}$, либо определено число $k_{1}\left(Z, \varphi_{r}, t_{0}, t_{1}\right)$. В последнем случае в обозначениях предыдущего параграфа положим $c=0, d=r, \psi(s) \equiv Z, \theta_{1} \equiv t_{0}, \theta_{2} \equiv t_{1}, \varphi(s, u)=\varphi_{s}(u)$ и либо

$(* *)$ найдется такая функция $z \in Z$, что $\pi(z)=\left[t_{0}, t_{1}\right]$ и $z\left(t_{0}\right)=z\left(t_{1}\right) \in K_{r}$, 
либо в силу утверждения $3.1 k_{1}\left(Z, \varphi_{r}, t_{0}, t_{1}\right)=k_{1}\left(Z, \varphi_{0}, t_{0}, t_{1}\right)$. Но число $k_{1}\left(Z, \varphi_{0}, t_{0}, t_{1}\right)$ равно нулю и, таким образом, отличие от нуля числа $k_{1}\left(Z, \varphi_{r}, t_{0}, t_{1}\right)$ означает вьполнение условия $(* *)$.

Значит, существование периодического решения будет установлено, если будет доказано, что $k_{1}\left(Z, \varphi_{r}, t_{0}, t_{1}\right) \neq 0$ при некотором $r>0$.

Теорема 4.1. Пусть выполнены условия $\mathrm{A}, Z \in R_{\text {сеһ }}(U)$ и условие 2) теоремы 1.1. Пусть $z\left(t_{0}\right) \neq z\left(t_{1}\right)$ для любой функиии $z \in Z$, удовлетворяюшей условиям $\pi(z)=\left[t_{0}, t_{1}\right] u z\left(t_{0}\right) \in S_{r} . \Pi y c m_{b} k_{1}\left(Z, \varphi_{r}, t_{0}, t_{1}\right) \neq 0, Y \in R_{c e h}(U) u \widetilde{\psi}_{i}(Y) \rightarrow Z n p u$ $i \rightarrow \infty$. Тогда найдется такая функиия $y \in Y$, что $\pi(y)=\left[t_{0}, t_{1}\right] u y\left(t_{0}\right)=y\left(t_{1}\right)$.

ДокАЗАТЕльство. Так как доказываемое свойство сохраняется при заменах переменных $\psi_{i}$, то его вьполнение достаточно доказать для одного из пространств $Y_{i}=\widetilde{\psi}_{i}(Y)$.

В силу теоремы 1.2 любая функция $z \in Y^{\mp}$, область определения которой содержит точку $t_{0}$, определена и на отрезке $\left[t_{0}, t_{1}\right]$. Это свойство сохраняется при заменах переменных $\psi_{i}$, т.е. любая функция $z \in Y_{i}^{\mp}$, область определения которой содержит точку $t_{0}$, определена и на отрезке $\left[t_{0}, t_{1}\right]$.

В силу наших предположений и теоремы $3.1 k_{1}\left(Y_{i}, \varphi_{r}, t_{0}, t_{1}\right) \neq 0$, начиная с некоторого $i$.

В силу замечаний начальной части этого параграффа это дает требуемое. Теорема доказана.

Теорема 4.2. Пусть $A$ - постоянная матрица $n$-го порядка и уравнение $y^{\prime}=A y$ не имеет периодических решений периода $\omega>0$, отличных от нулевого, функиия $f: \mathbb{R} \times \mathbb{R}^{n} \rightarrow \mathbb{R}^{n}$ измерима и периодична с периодом $\omega$ по первому аргументу (по “времени" $t \in \mathbb{R}$ ), непрерывна по второму аргументу (по "пространственному переменному" $y \in \mathbb{R}^{n}$ ) и существует такая локально интегрируемая по Лебегу неотрицательная функиия $\varphi(t)$, что $\|f(t, y)\| \leqslant \varphi(t)$ nри всех $t \in \mathbb{R}$ u y $\in \mathbb{R}^{n}$. Тогда уравнение $y^{\prime}=A y+f(t, y)$ имеет периодическое решение периода $\omega$.

ДокАЗАТЕЛЬСтво сводится к ссылке на теорему 4.1. При этом полагаем $t_{0}=0$, $t_{1}=\omega$. Роль пространства $Z$ играет пространство решений уравнения $y^{\prime}=A y$. Роль пространства $Y$ играет пространство решений уравнения $y^{\prime}=A y+f(t, y)$. Берем любую последовательность $\left\{\lambda_{i}: i=1,2, \ldots\right\}$, удовлетворяющую условию 2) теоремы 1.1.

Выполнение условий А очевидно.

Условия $Z \in R_{c e h}(U)$ и $Y \in R_{c e h}(U)$ также вьполнены (см. [1]).

Для подсчета индекса $k_{1}\left(Z, \varphi_{r}, t_{0}, t_{1}\right)$ воспользуемся обозначениями $\S 3$. Здесь $c=d$, $\theta_{1}(c)=t_{0}=0, \theta_{2}(c)=t_{2}=\omega$, отображение $q$ сопоставляет точке $u \in S_{r}$ вектор $z\left(t_{1}\right)-z\left(t_{0}\right)$, где $z$ - единственное решение задачи Коши

$$
z \in Z^{\mp}, \quad z\left(t_{0}\right)=u
$$

Так как отображение $q$ является ограничением на сферу невырожденного линейного преобразования $\mathbb{R}^{n} \rightarrow \mathbb{R}^{n}, y_{0} \in \mathbb{R}^{n}$, то $k_{1}\left(Z, \varphi_{r}, t_{0}, t_{1}\right)=+1$ или -1 . Мы доказали выполнение первого из условий в формулировке теоремы 4.1 .

Докажем вьполнение условия $\widetilde{\psi}_{i}(Y) \rightarrow Z$. Пусть $F(t)=\left\{u: u \in \mathbb{R}^{n},\|u\| \leqslant \varphi(t)\right\}$. Нам будет проще доказать, что $\widetilde{\psi}_{i}(\widetilde{Y}) \rightarrow Z$, где $\widetilde{Y}$ - пространство решений дифференциального включения $y^{\prime}=A y+F(t)$. В силу включения $Y \subseteq \widetilde{Y}$ отсюда будет следовать, 


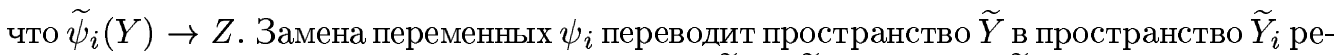
шений включения $y^{\prime}=A y+\lambda_{i} F(t)$. При этом $\widetilde{Y}_{1} \subseteq \widetilde{Y}_{2} \subseteq \cdots и \bigcap\left\{\widetilde{Y}_{i}: i=1,2, \ldots\right\}=Z$. Сходимость $\widetilde{Y}_{i} \rightarrow Z$ следует теперь из [5, теорема 2].

Продолжая по периодичности решение, существование которого установлено в теореме 4.1, получаем требуемое. Теорема доказана.

Заметим, что теорему 4.2 нельзя доказать с обычным в таких ситуациях использованием метода направляющих функций (см. [1]), так как возмущающий член в любой отдельно взятой точке может быть каким угодно. По тем же соображениям нельзя воспользоваться и другими прямыми методами подсчета индекса $k_{1}$ в обход использованного в $\oint 3$ предельного перехода. Теорема 4.2 распространяется и на дифференциальные включения, а именно справедлива

Теорема 4.3. Пусть $A$ - постоянная матрица $n$-го порядка и уравнение $y^{\prime}=A y$ не имеет периодических решений периода $\omega>0$, отличных от нулевого. Пусть многозначное отображсение $F: \mathbb{R} \times \mathbb{R}^{n} \rightarrow \mathbb{R}^{n}$ удовлетворяет следующим условиям:

отобрахсение $F(t, y)$ периодично с периодом $\omega$ по первому аргументу, его значения непусты, выпукль и компактны, отображсение $F(t, y)$ полунепрерывно сверху по второму аргументу, для любого $у \in \mathbb{R}^{n}$ существует такая измеримая функиия $f: \mathbb{R} \rightarrow \mathbb{R}^{n}$, что $f(t) \in F(t, y)$ при всех $t \in \mathbb{R}$, существует такая локально интегрируемая по Лебегу неотрицательная функиия $\varphi(t)$, что $F(t, y) \subseteq\left\{u: u \in \mathbb{R}^{n},\|u\| \leqslant \varphi(t)\right\}$ при всех $t \in \mathbb{R} u y \in \mathbb{R}^{n}$.

Тогда уравнение $y^{\prime}=A y+f(t, y)$ имеет периодическое решение периода $\omega$.

Доказательство этой теоремы повторяет доказательство теоремы 4.2 , непосредственньм обобщением которой является теорема 4.3 .

Следующие ниже замечания также аналогичным образом легко переносятся на дифференциальные включения, но для простоты обозначений и восприятия мы ограничимся случаем уравнений.

Теорема 4.4. Пусть $A$ - постоянная матрица $n$-го порядка и уравнение $y^{\prime}=A y$ не имеет периодических решений периода $\omega>0$, отличных от нулевого, $0 \leqslant \alpha<1$ и функиия $f: \mathbb{R} \times \mathbb{R}^{n} \rightarrow \mathbb{R}^{n}$ измерима и периодична с периодом $\omega$ по первому аргументу, непрерывна по второму аргументу и существует такая локально интегрируемая по Лебегу неотрицательная функция $\varphi(t)$, что $\|f(t, y)\| \leqslant(1+\|y\|)^{\alpha} \varphi(t)$ при всех $t \in \mathbb{R}$ и $y \in \mathbb{R}^{n}$. Тогда уравнение $y^{\prime}=A y+f(t, y)$ имеет периодическое реиение периода $\omega$.

ДоКАЗАТЕЛЬСТВо в основном повторяет доказательство теоремы 4.2, но с заменой $F(t)$ на $F(t, y)=\left\{u: u \in \mathbb{R}^{n},\|u\| \leqslant(1+\|y\|)^{\alpha} \varphi(t)\right\}$.

Замена переменных $\psi_{i}$ переводит пространство $\widetilde{Y}$ решений включения $y^{\prime}=A y+F(t, y)$ в пространство $\widetilde{Y}_{i}$ решений включения $y^{\prime}=A y+F_{i}(t, y)$, где $F_{i}(t, y)=\left\{u: u \in \mathbb{R}^{n}\right.$, $\left.\|u\| \leqslant \lambda_{i}\left(1+\|y\| / \lambda_{i}\right)^{\alpha} \varphi(t)\right\}$. Так как

$$
\lambda_{i}\left(1+\frac{\|y\|}{\lambda_{i}}\right)^{\alpha}=\lambda_{i}^{1-\alpha}\left(\lambda_{i}+\|y\|\right)^{\alpha} \rightarrow 0
$$

на любом шаре $\left\{u: u \in \mathbb{R}^{n},\|u\| \leqslant r\right\}$, то и здесь мы имеем требуемую сходимость. Теорема (с учетом замечаний доказательства теоремы 4.2) доказана. 
ПримеР. Покажем, как исследовать ситуацию при небольших отклонениях от условий приведенных теорем. Предположим выполненными предположения теоремы 4.4, зафиксируем любой вектор $u \in \mathbb{R}^{n}$ и для $y=\left(y_{1}, \ldots, y_{n}\right)$ положим

$$
g(t, y)=\|y\|^{\alpha}|\sin t|^{\sin \left(\ln \left|y_{1}\right|\right)+\left|y_{1}\right| /\|y\|-1},
$$

доопеделяя функцию $g$ нулем там, где написанная формула теряет смысл. Пусть для $M>0$

$$
g_{M}(t, y)=\min \{g(t, y), M\}
$$

Уравнение

$$
y^{\prime}=A y+f(t, y)+u g(t, y)
$$

не входит в зону действия приведенных теорем хотя бы потому, что нельзя указать интегрируемую мажоранту $\varphi\left(\right.$ если $y=\left(y_{1}, 0, \ldots, 0\right)$ и $\sin \left(\ln \left|y_{1}\right|\right)=-1$, то функция $g(t, y)$, с точностью до постоянных множителей, приобретает вид $\left.|\sin t|^{-1}\right)$.

Но множество особенностей $E$ (т.е. множество точек, где не выполняются локально предположения теоремы Каратеодори) устроено достаточно просто - оно счетно:

$$
\begin{aligned}
E=\{ & \left.\left(\pi k_{1},\left(e^{\pi k_{2}}, 0, \ldots, 0\right)\right): k_{1}, k_{2}=0, \pm 1, \pm 2, \ldots\right\} \\
& \cup\left\{\left(\pi k_{1},(0,0, \ldots, 0)\right): k_{1}=0, \pm 1, \pm 2, \ldots\right\},
\end{aligned}
$$

поэтому пространство решений $Y$ уравнения (9) принадлежит классу $R_{c}(U)$ (см. [2]-[4]). Уравнение же

$$
y^{\prime}=A y+f(t, y)+u g_{M}(t, y)
$$

локально удовлетворяет предположениям теоремы Каратеодори, и поэтому пространство его решений принадлежит классу $\left[R_{c e u}(U)\right]$.

При $M \rightarrow \infty$ пространство решений уравнений (10) сходится к пространству $Y$ (множество особенностей $E$ остается тем же самым, см. [2]-[4]), и поэтому предельное пространство $Y$ также принадлежит замкнутому множеству $\left[R_{c e u}(U)\right]$. По теореме 2.1 отсюда следует, что $Y \in R_{c e h}(U)$. Теперь, повторяя доказательства теорем 4.2 и 4.4 с $\lambda_{i}=e^{-\pi i}$, получаем существование периодического решения уравнения (10) периода $2 \pi$. 


\section{СПИСОК ЦИТИРОВАННОЙ ЛИТЕРАТУРЫ}

[1] Bielawski R., Górniewicz L., Plaskacz S. Topological approach to differential inclusions on closed subsets of $\mathbb{R}^{n} / /$ Dynam. Report. (N. S.) 1992. V. 1. P. 225-250.

[2] Филиппов В. В. Топологическое строение пространств решений обыкновенных дифференциальных уравнений // УМН. 1993. Т. 48. № 1. С. 103-154.

[3] Федорчук В. В., Филиппов В. В. Общая топология. Основные конструкции. М.: Изд-во МГУ, 1988.

[4] Филиппов В. В. Пространства решений обыкновенных дифференциальных уравнений. М.: Изд-во МГУ, 1993.

[5] Филиппов В. В. О теории задачи Коши для обыкновенного дифференциального уравнения с разрьвной правой частью // Матем. сб. 1994. Т. 185. №11. С. 95-118.

[6] Филиппов В. В. О семействах замен переменных и теореме единственности для обыкновенных дифференциальных уравнений // Докл. РАН. 1993. Т. 330. №6. С. 704-706.

[7] Энгелькинг Р. Общая топология. М.: Мир, 1986.

[8] Górniewicz L. Topological approach to differential inclusions // Topological methods in differential equations and inclusions. Ser. C. V. 472: Kluwer Acad. Publ., 1995. P. 129-190.

[9] Frigon M. Théorèmes d'existence de solutions d'inclusions différentielles // Topological methods in differential equations and inclusions. Ser. C. V. 472: Kluwer Acad. Publ., 1995. P. 51-88. 